\title{
Vimentin 3, the New Hope, Differentiating RCC versus Oncocytoma
}

\author{
Melanie von Brandenstein, ${ }^{1}$ Katharina Puetz, ${ }^{1}$ Monika Schlosser, ${ }^{1}$ \\ Heike Löser, ${ }^{1}$ Joachim P. Kallinowski, ${ }^{2}$ Daniel Gödde, ${ }^{3}$ Reinhard Buettner, ${ }^{1}$ \\ Stefan Störkel, ${ }^{3}$ and Jochen W. U. Fries ${ }^{1}$ \\ ${ }^{1}$ Institute of Pathology, University Hospital of Cologne, Kerpenerstraße 62, 50924 Cologne, Germany \\ ${ }^{2}$ Institute of General, Visceral and Minimal Invasive Surgery, Clinic Northwest, Steinbacher Hohl 2-26, \\ 60488 Frankfurt am Main, Germany \\ ${ }^{3}$ Institute of Pathology, Helios Clinic Wuppertal, University Clinic Witten-Herdecke, Heusnerstraße 40, 42283 Wuppertal, Germany
}

Correspondence should be addressed to Jochen W. U. Fries; jochen.fries@uni-koeln.de

Received 24 October 2014; Accepted 3 March 2015

Academic Editor: Claudio Letizia

Copyright (C) 2015 Melanie von Brandenstein et al. This is an open access article distributed under the Creative Commons Attribution License, which permits unrestricted use, distribution, and reproduction in any medium, provided the original work is properly cited.

\begin{abstract}
Vimentin is currently used to differentiate between malignant renal carcinomas and benign oncocytomas. Recent reports showing Vimentin positive oncocytomas seriously question the validity of this present diagnostic approach. Vimentin 3 is a spliced variant and ends with a unique $\mathrm{C}$-terminal ending after exon 7 which differentiates it from the full length version that has 9 exons. Therefore, the protein size is different; the full length Vimentin version has a protein size of $\sim 57 \mathrm{kDa}$ and the truncated version of $\sim 47 \mathrm{kDa}$. We designed an antibody, called Vim3, against the unique C-terminal ending of the Vimentin 3 variant. Using immune histology, immune fluorescence, Western blot, and qRT-PCR analysis, a Vim3 overexpression was detectable exclusively in oncocytoma, making the detection of Vim3 a potential specific marker for benign kidney tumors. This antibody is the first to clearly differentiate benign oncocytoma and the mimicking eosinophilic variants of the RCCs. This differentiation between malignant and benign RCCs is essential for operative planning, follow-up therapy, and patients' survival. In the future the usage of Vimentin antibodies in routine pathology has to be applied with care. Consideration must be given to Vimentin specific binding epitopes otherwise a misdiagnosis of the patients' tumor samples may result.
\end{abstract}

\section{Introduction}

An oncocyte is an epithelial cell characterized by an excessive amount of mitochondria. Hamperl named them in 1931 after the Greek word "onkousthai" (to swell) and first described them as a distinct cell system consisting of large epithelial cells with irregular nuclei and finely granular, acidophilic cytoplasm [1]. The fundamental morphological nature of oncocytes, an abundance of mitochondria, was firmly established by electron microscopy [2]. Since then oncocytes have been detected in various organs (i.e., thyroid, parathyroid, and salivary glands) as well as in different tumors (i.e., oncocytomas, Hürthle cell tumors of the thyroid, oxyphilic adenoma of parathyroid gland, and Warthin's tumor of salivary gland) (encyclopedia of Biol Chem 2004).

Renal oncocytomas, initially identified by Zippel, in 1942 [3], have been regarded as predominantly benign renal neoplasms since the first study by Klein and Valensi [4], although occasional reports of malignant cases have been reported [5]. The major diagnostic problem is the differential to other renal tumors: (i) the eosinophilic or granular variant of clear cell renal carcinoma (RCC) and (ii) the chromophobe RCC. Differential diagnosis currently uses immune histology to differentiate malignant renal cell carcinoma from oncocytoma. For chromophobe carcinoma, positivity for claudin 8 and negativity for claudin 7 have been shown 
as the characteristic constellation [6]. To differentiate the chromophobe and eosinophilic RCC from oncocytoma, the positivity for Vimentin, a structural protein, has been used to identify the former [7]. However, a series of oncocytomas has recently been reported in which a Vimentin positivity has been observed, making the differentiation questionable, particularly in preoperative evaluation [8]. Hes et al. analyzed 234 oncocytoma of which $73 \%$ were positive for Vimentin staining [8]. Vimentin is an intermediate-sized filament that functions in cellular signal transduction, structural integrity of cells and tissues, and adhesion and migration [9]. In 2007 a spliced variant of Vimentin with a unique C-terminal ending was detected by a working group at the Craig Venter Institute (NHLBI Resequencing and Genotyping Service (RSG), N01-NV-48196, J. Craig Venter Institute, Rockville, MD 20850) and published online in PubMed (Accession number ACA06103.1). In 2011 Thakkar et al. [10] described the presence of this variant in gliomas. However, no further analysis or investigation regarding its role has been performed.

Based on the knowledge that the spliced variant of Vimentin is 35 amino acids smaller than the full length variant, we compared both sequences with the detailed information of the Vimentin 3B4 antibody. From the literature it is known that the 3B4 Vimentin antibody detects the rod domain [11] which is a homologue to the truncated Vimentin variant 3 (Vim3) rod domain. Thus, it seemed possible that the protein expression of Vimentin described in the literature by immune histology resulted from the combined detection not only of the protein from full length, but also of the spliced variant of Vimentin, namely, Vim3.

Most of the commercially available antibodies (clones 3B4 and SP20) are against epitopes located in the rod domain of Vimentin (Figure 1). The clone V9 is directed against the taildomain of Vimentin. However, for the detection of the truncated Vimentin variant 3 (Vim3), the Vim3 antibody is used, which is designed against the unique $\mathrm{C}$-terminal ending.

In case of renal tumors with eosinophilic apperance, which mimic oncocytoma, the differential diagnosis between RCCs and oncocytomas is based on a panel of different antibodies. In particular the presence or absence of Vimentin staining of paraffinized tumor samples can be of great importance for the differentiation between malignant and benign tumors.

This diagnostic approach has to be reevaluated, since a spliced Vimentin isoform exists. This is also detectable with the currently used antibodies against the $\mathrm{N}$-terminal sequence; thus Vimentin positivity is no longer a diagnostic feature per se of malignant RCCs. Thus, in this paper we analyzed the presence of Vim 3 versus the full length Vimentin in RCCs, especially the eosinophilic variant of RCCs versus oncocytomas.

We designed primers which detect either the full length version of Vimentin or its spliced variant, Vim3. After performing a qRT-PCR on paraffin embedded tissues of the different RCCs and oncocytoma we could indeed show that Vim3 is the predominant variant in oncocytoma. Furthermore, we designed an antibody exclusively detecting the unique C-terminus of Vim3.

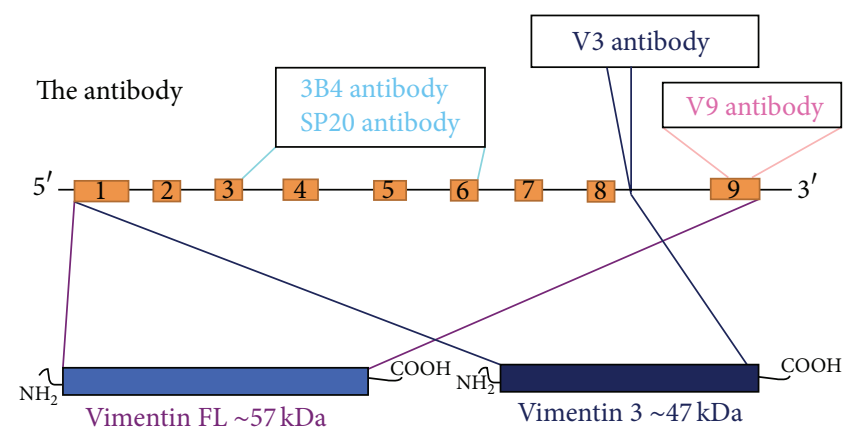

FIGURE 1: Differential location of the different antibodies against Vimentin. The commercially available antibodies of the clones 3B4 and SP20 were against the rod domain (slight blue); the antibody clone V9 is against C-terminal ending of the full length variant (slight purple); and the truncated antibody against the unique Cterminal ending Vim3 is indicated with dark blue.

This is the first report describing the presence and the structural differences of Vim3 versus the full length Vimentin. Our data present strong evidence that Vim 3 is the isoform responsible for the so-called Vimentin positive oncocytomas described in the literature. Furthermore, we show that the V9 Vimentin antibody as well as antibodies detecting the full length version of Vimentin cannot be used any longer for differential diagnosis between RCCs and oncocytomas, because these result in misdiagnoses with potentially grave consequences for the patients involved.

\section{Materials and Methods}

2.1. Antibody Design and Quantification. The Vim3 antibody was commercially designed (EZbiolab, Inc.) using the unique C-terminal ending of Vim3 as target (for detailed information please see patent by University of Cologne, Brandenstein/Fries, patent number EP 13160876.2-1405). The Vim3 expression versus that of full length Vimentin (clone V9) (Santa Cruz, Heidelberg) was shown using immune histology on paraffin embedded colon mucosa biopsies from our pathology archive. Western blot analysis (see below) of macrodissected material of cryptal epithelial cells and lymphoid cells was performed for further evaluation and proof of specificity of the newly designed antibody.

2.2. Immune Fluorescence of Paraffin Embedded Tissues. $4 \mu \mathrm{m}$ thick paraffin embedded tissue sections were deparaffinized by incubation for $1 \times 10 \mathrm{~min}$ in xylene, followed by $1 \times 5 \mathrm{~min}$ $100 \%$ ethanol and $1 \mathrm{~min} 70 \%$ ethanol, and then rinsed with distilled water. The slides were digested with Proteinase $\mathrm{K}$ for $30 \mathrm{~min}$ at room temperature. After an incubation period in $5 \%$ PBS milk for $30 \mathrm{~min}$, the slides were reincubated for 1 hour at room temperature with specific primary antibodies (Vim3) in 3\% PBS milk. Following washes with PBS, the sections were incubated with a secondary FITC-anti-rabbit antibody (Santa Cruz). Subsequent to rinsing with PBS, the slides were then counterstained with DAPI mounting medium (nuclear staining) and cover slipped. 
2.3. Immune Histology of Paraffin Embedded Tissues. Paraffin embedded tissue sections ( $4 \mu \mathrm{m}$ thick) were deparaffinized by incubation for 2-5 minutes in xylene, followed by 2-3 minutes in $100 \%$ ethanol and 1 minute in $95 \%$ ethanol, and then rinsed with distilled water. The slides were incubated with a specific serum blocker (anti-rabbit) for 30 minutes in order to avoid nonspecific binding. After that incubation period, the slides were reincubated for 1 hour at room temperature with specific primary antibodies (Vim3, EZBiolab, Inc. Carmel, USA, Vimentin V9, Santa Cruz, Heidelberg, Germany, AMACR and CD117 [12], Dako, Hamburg, Germany). Following washes with PBS-Tween 20, the sections were incubated with a secondary, anti-rabbit antibody (Santa Cruz, Heidelberg, Germany). After rinsing with PBS-Tween 20, the slides were reincubated for 2 minutes in 95\% ethanol, followed by 2-3 minutes in $100 \%$ methanol, counterstained with $\mathrm{H} \& \mathrm{E}$, and cover slipped.

The analyzed paraffin embedded tissue sections were from retrospective nephrectomies; nevertheless we performed a blind study, so any bias of the results could be excluded.

2.4. Oncocytic Tumors. Since human materials were used, procedures were followed as outlined in accordance with ethical standards formulated in the Declaration of Helsinki 1975, with preapproval by the Ethics Committee at the University Hospital, Cologne, Germany (reference number 09-232).

2.5. Quantitative Real-Time PCR ( $q R T-P C R)$. The qRT-PCR was performed as previously described $[13,14]$.

For quantitative analysis, $\beta$-actin was measured. All samples were normalized to $\beta$-actin as the reference gene. All experiments were performed in triplicate. Relative fluorescence was calculated using the $\Delta \Delta$-CT method, as outlined in User Bulletin 2 (PE Applied Biosystems, Darmstadt, Germany). The statistical significance of $\mathrm{qPCR}$ values at different time points was assessed by Student's paired $t$-test. Table 2 provides primer information.

2.6. RNA-Extraction Paraffin Embedded Tissues and RT$P C R$. Formalin-fixed and paraffinized (FFPE) human tissue samples, from the archives of the Department of Pathology, University Hospital of Cologne, Cologne, Germany, and the Department of Pathology, Helios Clinic Wuppertal, University Clinic Witten-Herdecke, Wuppertal, Germany, were used.

RNA extraction from FFPE tissue was performed according to the RNeasy FFPE kit (Qiagen, Germany). RNA quantification was accomplished using NanoDrop technology.

The cDNA was obtained from 250 ng of RNA using random primers and SuperScript III reverse transcriptase, according to the manufacturer's protocol (Invitrogen, Darmstadt, Germany).

2.7. Statistical Analysis. For statistical analysis the GraphPad Prism 5 program was used. Analysis of variance (ANOVA) was performed and the significant differences were calculated and indicated by stars $\left({ }^{*} P<0.05,{ }^{* *} P<0.01\right.$, and $\left.{ }^{* * *} P<0.001\right)$. All differences without indication were not statistically significant.

2.8. Western Blot. All Western blots were performed in triplicate as outlined in detail before (Gerstung et al. [13]). $\beta$-actin served as loading control (Santa Cruz, Heidelberg, Germany). The Vimentin 3 antibody was used in a 1:500 dilution, and the V9 antibody (Santa Cruz) against full length Vimentin was employed in 1:1000 as recommended by the supplier. Protein extraction from paraffinized tissue was done as described in Ikeda et al. [15]. The $4 \mu \mathrm{m}$ paraffinized tissue samples were incubated in Xylol for $15 \mathrm{sec}$, mixed, and then centrifuged for $2 \mathrm{~min}$ at full speed and at room temperature. $100 \%$ ethanol was added to the pellet for $2 \mathrm{~min}$, then mixed, and again centrifuged for $2 \mathrm{~min}$ at full speed and at room temperature. After carefully discarding the supernate, the pellet was air dried. $50 \mu \mathrm{L}$ of RIPA buffer was added, incubated at $100^{\circ} \mathrm{C}$ for $20 \mathrm{~min}$, and then followed by an incubation period of 2 hours at $60^{\circ} \mathrm{C}$. The samples were subsequently centrifuged at full speed at $4^{\circ} \mathrm{C}$ for $20 \mathrm{~min}$. The supernate was then stored at $-80^{\circ} \mathrm{C}$ until further use. Protein quantification was performed as previously described [13].

\section{Results}

3.1. Antibody Evaluation. Since Vimentin is commonly known primarily as a mesenchymal marker, we characterized the Vim 3 antibody using frozen sections of appendiceal tissue containing epithelial, mesenchymal, and lymphatic tissue elements. As Figure 2 shows, Vim3 was expressed in colonic crypt epithelium, particularly in the regeneratively active part of the crypt, in mesenchymal cells, and in lymphocytes. A Western blot was performed to verify the expected size of the Vim3 splice form being $47 \mathrm{kDa}$ (Figure 2), while the full length molecule was predictably $57 \mathrm{kDa}$ (data not shown).

We also established the Vim3 antibody binding pattern in renal tissues. The Vimentin full length molecule was evident in different types of mesenchymal cells (such as fibroblasts and smooth muscle cells) and also in proximal tubule cells.

3.2. mRNA Detection of Vimentin and Vim3. The full length molecule of Vimentin is used as a marker to differentiate benign oncocytomas, expected to be negative, from malignant renal cell carcinomas being Vimentin positive. Our qRTPCR evaluation of renal tumors confirmed this finding in cases from the pathology archives, while demonstrating that full length Vim3 was expressed in (Table 1) Onco (Oncocytoma). RCC subtypes express lower levels of Vim3 mRNA.

3.3. Protein Detection of Vim3 versus Full Length Vimentin in RCCs. By immune histology on paraffinized tissue slices from renal tumors, full length Vimentin protein was found to be strongly expressed in clear cell RCCs and papillary RCCs. Chromophobe RCCs showed a weak reactivity with the antibody, while oncocytomas demonstrated no reactivity. In contrast, Vim3 expression was strong in oncocytomas, while all three malignant RCCs subtypes were negative (Figure 4). 


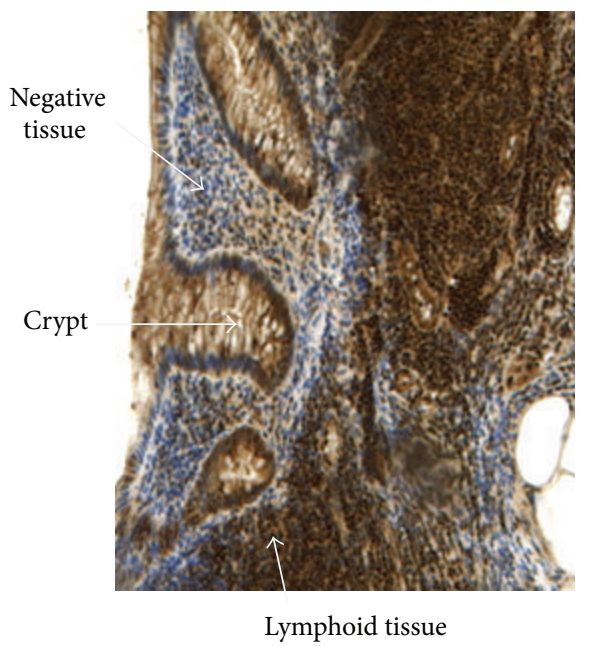

(a)

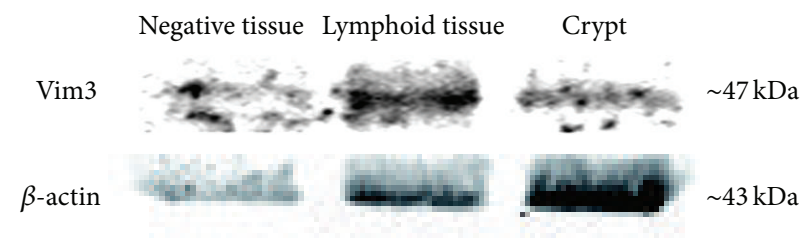

(b)

FIGURE 2: Evaluation of Vim3 antibody. (a) Immune histology showing expression of Vim3 in colonic crypt epithelium and in lymphocytes. (b) Western blot analysis after macrodissection of crypt epithelium and lymphocytes. $\beta$-actin serves as loading control.

TABLE 1: Tumor types and patient number.

\begin{tabular}{lc}
\hline Patient number & Diagnosis \\
\hline $1-6$ & Normal kidney control \\
$7-22$ & Oncocytoma \\
$23-33$ & Chromophobe RCC \\
$34-44$ & Papillary RCC \\
$45-54$ & RCC \\
$55-60$ & Eosinophilic RCC \\
\hline
\end{tabular}

Consequently, using immune fluorescence analyses of the different RCC subtypes and the oncocytoma a clear expression of Vim3 was only detectable in oncocytoma. The oncocytoma mimicking variant of RCC, namely, the eosinophilic variant, was negative for Vim3 (Figure 5).

3.4. Collision Tumor. To further demonstrate the applicability of the new Vim3 antibody, a collision tumor consisting of two different tumor subtypes was used. Figure 6 shows the $H \& E$ staining of its papillary RCC differentiation. Since the patient suffered from pleural metastases, it was important to identify their origin. Therefore, we performed an immune fluorescence staining for Vimentin FL positive (V9) and for Vim3. This indicated that the first tumor type with V9 being positive and Vim3 being negative was the malignant component. The histogenesis of the second tumor type found in the tissue sample was questionable, possibly being a "real" oncocytoma. After immune fluorescence for Vimentin FL (V9) as well as for Vim3 was performed, only the Vim3 staining showed positive areas, indicating that the second tumor type was indeed an oncocytoma.

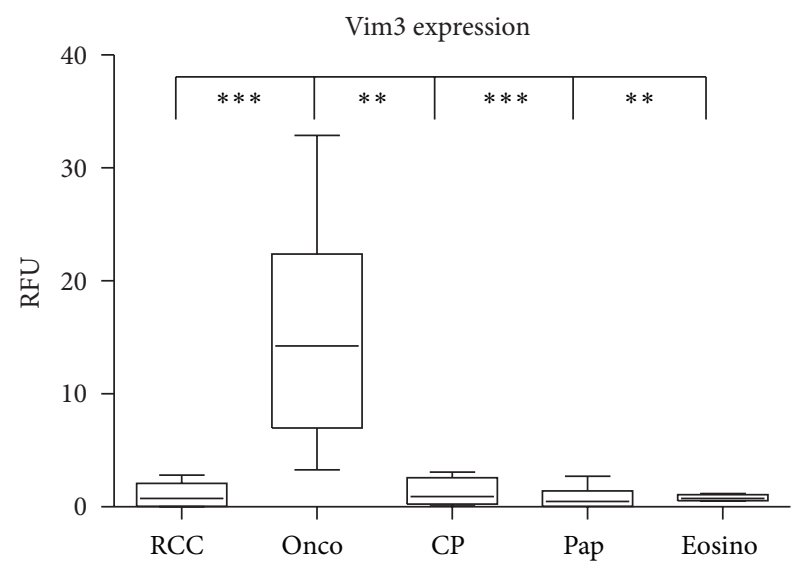

FIGURE 3: qRT-PCR analysis for Vim3 in oncocytoma versus clear cell renal cell carcinoma (RCC), chromophobe RCC (CP), papillary RCC (Pap), and eosinophilic RCC (Eosino). $\beta$-actin was used as reference gene. ${ }^{*} P<0.05 ;{ }^{* *} P<0.01 ;{ }^{* *} P<0.001$, and all differences without indication are not statistically significant.

\section{Discussion}

In this paper, we characterize a Vimentin splice isoform, called Vimentin 3 (Vim3), as a potentially important structural cellular protein. Its unique structure leads to a $10 \mathrm{kDa}$ smaller protein (Figure 1), which is more widely expressed than its full length counterpart, particularly in epithelial cells and lymphocytes (Figure 2).

To study the importance of Vim 3 for renal tubule cells further, we analyzed Vim3 versus full length Vimentin expression by qRT-PCR in renal tumors (Figure 3). Surprisingly, while RCCs have high amounts of transcribed full length Vimentin, they are almost Vim3 negative. In contrast, the reverse is true for oncocytomas: while their negativity for 
TABle 2: Primers.

\begin{tabular}{|c|c|c|c|}
\hline Gene & Sequence & Annealing temp. & Cycles \\
\hline$\beta$-actin & $\begin{array}{l}\text { Forw. 5'-TTGGCAATGAGCGGTTCCGCTG-3' } \\
\text { Rev. 5' -TACACGTGTTTGCGGATGTCCAC-3' }\end{array}$ & $55^{\circ} \mathrm{C}$ & $40 \mathrm{x}$ \\
\hline Vimentin, full length & $\begin{array}{l}\text { Forw. } 5^{\prime} \text {-GAGAACTTTGCCGTTGAAGC-3' } \\
\text { Rev. } 5^{\prime} \text {-TCCAGCAGCTTCCTGTAGGTG-3 }{ }^{\prime}\end{array}$ & $55^{\circ} \mathrm{C}$ & $40 \mathrm{x}$ \\
\hline Vim3 & $\begin{array}{l}\text { Forw. } 5^{\prime} \text {-GAGAACTTTGCCGTTGAAGC-3' } \\
\text { Rev. } 5^{\prime} \text {-GAAATAAAATGCTTACCCCTCAG-3' }\end{array}$ & $55^{\circ} \mathrm{C}$ & $40 \mathrm{x}$ \\
\hline
\end{tabular}

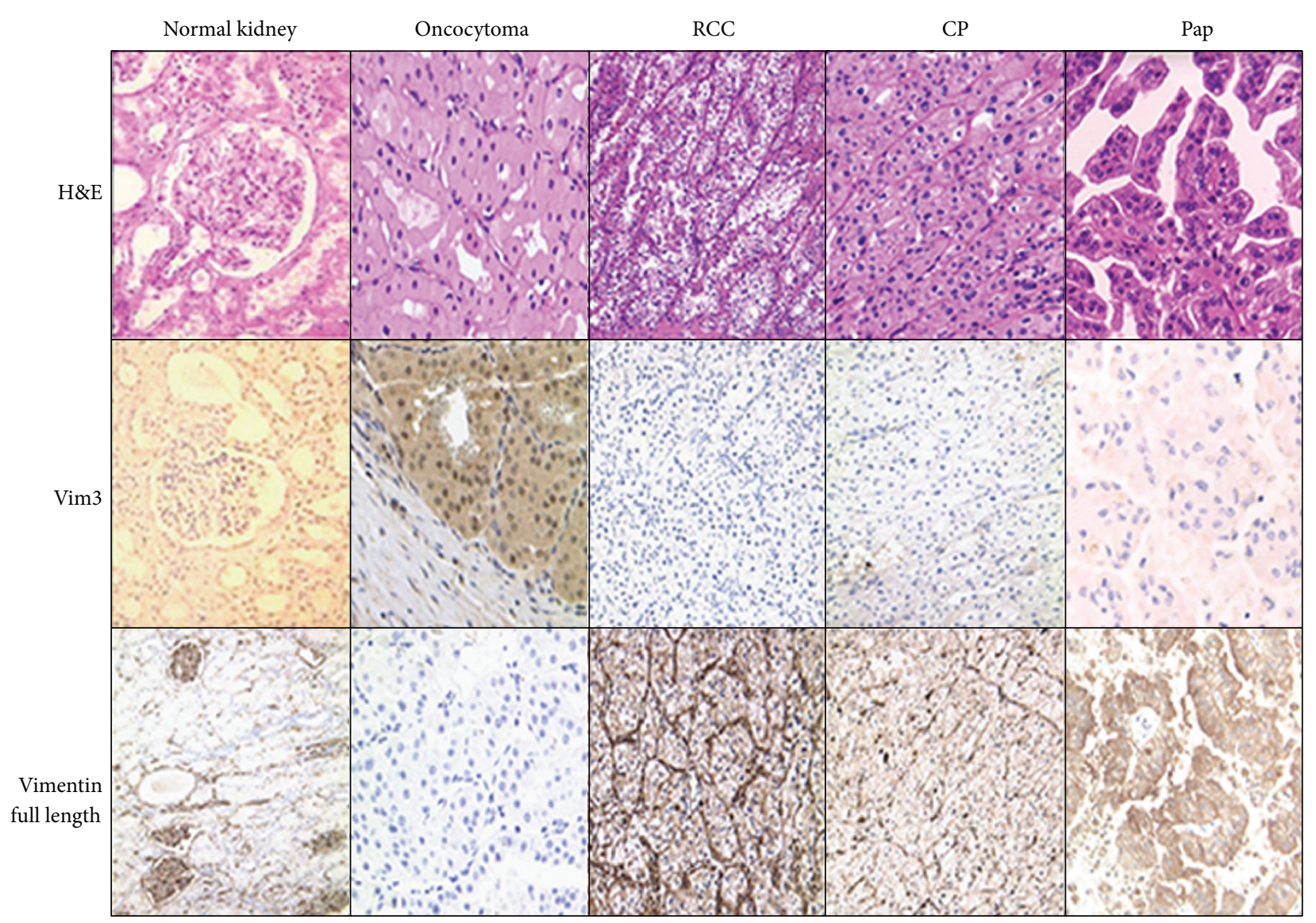

FiguRE 4: Immune histological analysis of the expression pattern between oncocytoma and RCC subtypes. Full length Vimentin positive tumor cells are observed in clear cell and papillary RCCs, while Vim3 positive cells are only found in oncocytoma, which otherwise are negative for full length Vimentin. H\&E staining of typical tumor morphology.

full length Vimentin is not surprising (and being a criteria for their identification), the levels for Vim3 are unexpectedly high. The papillary RCC subtype (Pap) has small mRNA level of Vimentin full length and Vim3 detectable by qRT-PCR.

Nevertheless, due to some posttranscriptional modifications, the Vim3 signal is not detectable by immune histology (Figure 4), while a strong Vimentin (full length) staining can be easily detected. The only positive signal, regarding the Vim3, was detectable in case of oncocytoma, and the "normal" tissue section was negative for Vim3 (Figure 4).

This result as well as the immune fluorescence results (Figure 5) identifies Vim3 as potential immune histology marker for renal oncocytomas.
Currently, it is still common practice in routine pathology to differentiate renal cell carcinomas from carcinomas of histogenetically different origins by using immune histology with cytokeratins and Vimentin. In particular, Vimentin positivity has been regarded as the major hallmark not only for RCC but also for differentiating them from their benign counterparts, the oncocytomas. Since Hes et al. [8] reported Vimentin positivity in $73 \%$ of all tested oncocytomas, this diagnostic approach has been questionable, while its underlying mechanism has been elusive.

From our results, we claim that by using an antibody against the unique C-terminal sequence of Vim3 "real" oncocytomas can be unequivocally identified. 


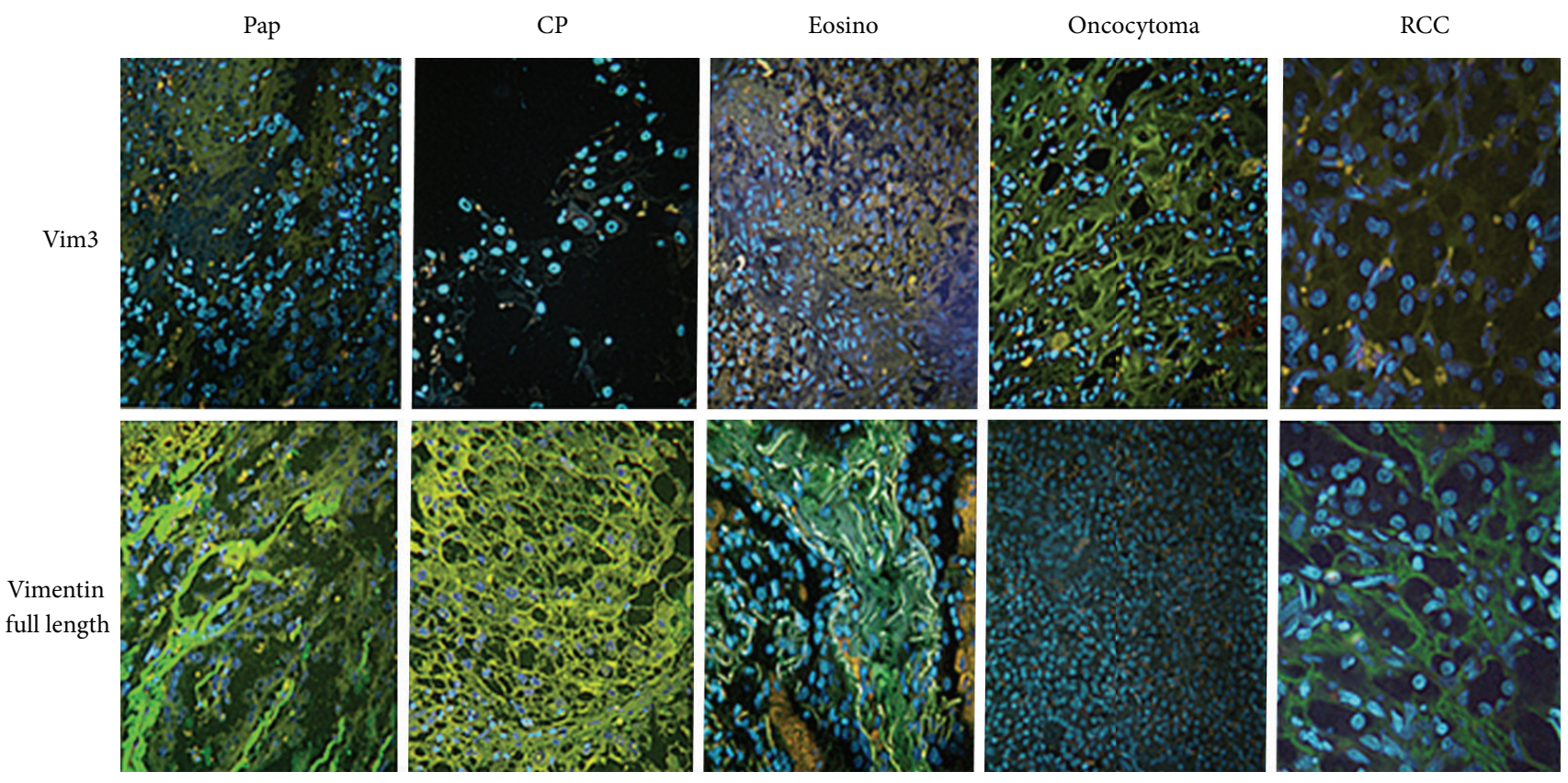

FIGURE 5: Immune fluorescence of oncocytoma and RCC subtypes. Full length Vimentin positive tumor cells are observed in clear cell and papillary RCCs, while Vim3 positive cells are only found in oncocytoma, which otherwise are negative for full length Vimentin. H\&E staining of typical tumor morphology; original magnification $\times 400$.

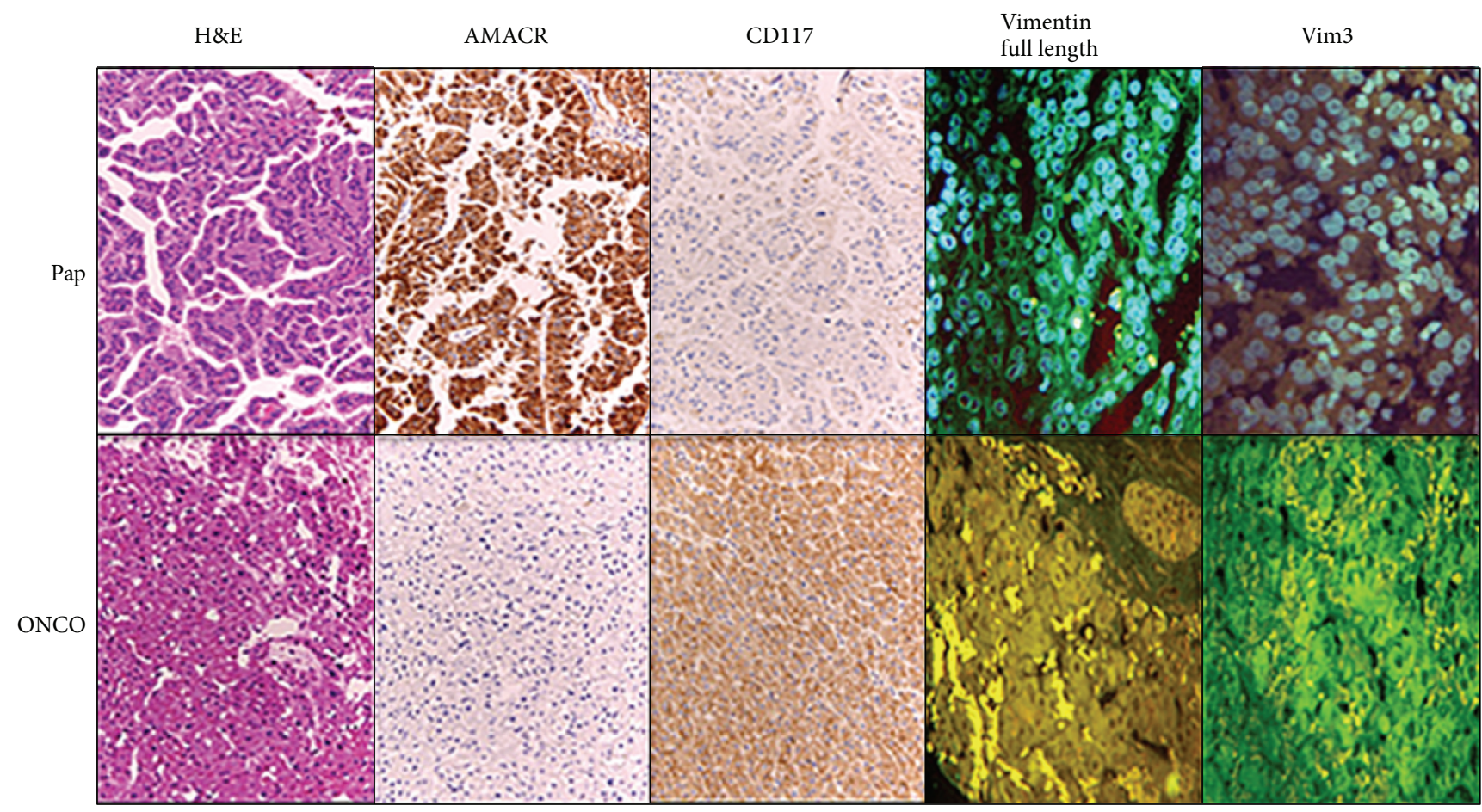

FIGURE 6: Collision tumor, with two different tumor subtypes. Top row: papillary RCC; bottom row: preliminary diagnosis, oncocytoma. H\&E staining and immunohistological staining for AMACR and CD117, Vimentin FL, and Vim3 were performed. Questionable was the second tumor type unexpectedly found in the tissue sample. Immune fluorescence staining for Vimentin FL positive (V9) was positive and the immune fluorescence for Vim3 was negative in the papillary RCC component, indicating the malignant tumor subtype, whereas the second one is an oncocytoma (Vim3 positive). 
The exact nature and mechanism of the "Vimentin positive oncocytomas" require further clarification. Our results indicate that these tumors have to be classified as an eosinophilic variant of clear cell RCCs. Since their morphologic appearance on an $\mathrm{H} \& \mathrm{E}$ slide seems identical to a "true" oncocytoma, we performed an immune fluorescence for Vim3 (Figure 5). This resulted in a clearly Vim3 negative appearance of these tumors regarded as "true" oncocytomas.

However, the importance of this study for routine pathologic diagnoses with respect to the mystery of "Vimentin positive oncocytomas" advocates our current explanation.

To date, an intracellular role of Vim3 has not been defined while an intracellular role of the full length Vimentin molecule has been described in the literature as an anchoring molecule for the nucleus [16]. Knowing the interaction of its full length counterpart, one may speculate about Vim3's intracellular importance. Since the N-terminal domain and the rod domain have not changed, binding partners such as ankyrin [17] and interactions with plectin [18] should still be possible. In contrast, the missing tail and the unique amino acids of its C-terminal ending may result in differences in the C-terminal interaction. Currently, the tail-domain has been reported to be the binding and interactive site for $\mathrm{F}$ actin [19] and lamin B [17]. However, since the major part of the C-terminus is absent in Vim 3 and the exact interaction sites for both molecules are presently unknown, further investigations have to be conducted in order to fully elucidate potential interaction, or its absence, between Vim3 and other structural binding partners. This protein differentiates benign oncocytoma from malignant RCC variants, especially the eosinophilic RCC variant, which mimics oncocytoma by immune histology and immune fluorescence.

To strengthen our interpretation, we applied the Vim3 antibody to a collision tumor (Figure 6), in which we identified its metastatic component as belonging to the papillary differentiation being Vim 3 negative, while the other part was identified as an oncocytoma, based on its Vim3 positivity. CD117 expression is a hallmark in differentiating oncocytoma and chromophobe RCC [20]. In case of the examined collision tumor, CD117 positivity could be a problem, since the chromophobe RCC is a malignant tumor and the oncocytoma is a benign one, so the differentiation of the metastatic component of the collision tumor is still questionable (Figure 6). The further usage of an alpha-methyl CoA racemase (AMACR) antibody can be used as well for distinction between chromophobe RCC and oncocytoma [21]. AMACR positivity is seen in papillary RCC [22] and can be used as marker between primary and metastatic RCC [21].

Figure 6 shows the two tumor types, and a clear differentiation between the RCC subtypes, namely, the papillary part of the tumor and the benign part, was possible due to the usage of our Vim3 antibody.

In conclusion, we present here a unique Vimentin isoform, Vim3, as a differential marker between malignant RCCs and oncocytoma. We strongly believe that this clear differentiation between the benign and malignant kidney tumor types will be essential in the future for patients' therapy as well as operative planning, follow-up therapy, and patients' survival.

\section{Conflict of Interests}

The authors declare no conflict of interests.

\section{Acknowledgments}

Melanie von Brandenstein was supported by Koeln Fortune Program/Faculty of Medicine, University of Cologne, a recipient of a postdoctoral fellowship and excellence cluster initiative supported by University of Cologne and DFG. The authors gratefully acknowledge the support by Susanne Sattler in immune histology, Dr. Heike Göbel for her help with the antibody quantification and critical reading of the paper, and Claudia Richter for her excellent technical assistance.

\section{References}

[1] H. Hamperl, "Über das Vorkommen von Onkocyten in verschiedenen Organen und ihren Geschwülsten-Mundspeicheldrüsen, Bauchspeicheldrüse, Epithelkörperchen, Hypophyse, Schilddrüse, Eileiter,' Virchows Archiv für Pathologische Anatomie und Physiologie und für Klinische Medizin, vol. 298, no. 2, pp. 327-375, 1936.

[2] R. Kataoka, Y. Hyo, T. Hoshiya, H. Miyahara, and T. Matsunaga, "Ultrastructural study of mitochondria in oncocytes," Ultrastructural Pathology, vol. 15, no. 3, pp. 231-239, 1991.

[3] J. Zippel, “Zur Kenntnis der Onkocyten," Virchows Archiv, vol. 308, no. 2, pp. 360-382, 1942.

[4] M. J. Klein and Q. J. Valensi, "Proximal tubular adenomas of kidney with so-called oncocytic features. A clinicopathologic study of 13 cases of a rarely reported neoplasm," Cancer, vol. 38, no. 2, pp. 906-914, 1976.

[5] J. D. Oxley, J. Sullivan, A. Mitchelmore, and D. A. Gillatt, "Metastatic renal oncocytoma," Journal of Clinical Pathology, vol. 60, no. 6, pp. 720-722, 2007.

[6] A. O. Osunkoya, C. Cohen, D. Lawson, M. M. Picken, M. B. Amin, and A. N. Young, "Claudin-7 and claudin-8: immunohistochemical markers for the differential diagnosis of chromophobe renal cell carcinoma and renal oncocytoma," Human Pathology, vol. 40, no. 2, pp. 206-210, 2009.

[7] R. Waldherr and K. Schwechheimer, "Co-expression of cytokeratin and vimentin intermediate-sized filaments in renal cell carcinomas: comparative study of the intermediate-sized filament distribution in renal cell carcinomas and normal human kidney," Virchows Archiv A, vol. 408, no. 1, pp. 15-27, 1985.

[8] O. Hes, M. Michal, N. Kuroda et al., "Vimentin reactivity in renal oncocytoma: immunohistochemical study of 234 cases," Archives of Pathology and Laboratory Medicine, vol. 131, no. 12, pp. 1782-1788, 2007.

[9] J. Ivaska, H.-M. Pallari, J. Nevo, and J. E. Eriksson, "Novel functions of vimentin in cell adhesion, migration, and signaling," Experimental Cell Research, vol. 313, no. 10, pp. 2050-2062, 2007.

[10] D. Thakkar, L. Shervington, and A. Shervington, "Proteomic studies coupled with RNAi methodologies can shed further light on the downstream effects of telomerase in glioma," Cancer Investigation, vol. 29, no. 2, pp. 113-122, 2011.

[11] M. Malakoutikhah, M. J. Gómara, J. A. Gómez-Puerta, R. Sanmartí, and I. Haro, "The use of chimeric vimentin citrullinated peptides for the diagnosis of rheumatoid arthritis," Journal of Medicinal Chemistry, vol. 54, no. 21, pp. 7486-7492, 2011. 
[12] P. H. Tan, L. Cheng, N. Rioux-Leclercq et al., "Renal tumors: diagnostic and prognostic biomarkers," The American Journal of Surgical Pathology, vol. 37, no. 10, pp. 1518-1531, 2013.

[13] M. Gerstung, T. Roth, H.-P. Dienes, C. Licht, and J. W. U. Fries, "Endothelin-1 induces NF- $\kappa$ B via two independent pathways in human renal tubular epithelial cells," American Journal of Nephrology, vol. 27, no. 3, pp. 294-300, 2007.

[14] M. G. von Brandenstein, A. N. Abety, R. Depping et al., "A p38p65 transcription complex induced by endothelin-1 mediates signal transduction in cancer cells," Biochimica et Biophysica Acta-Molecular Cell Research, vol. 1783, no. 9, pp. 1613-1622, 2008.

[15] K. Ikeda, T. Monden, T. Kanoh et al., "Extraction and analysis of diagnostically useful proteins from formalin-fixed, paraffinembedded tissue sections," Journal of Histochemistry and Cytochemistry, vol. 46, no. 3, pp. 397-403, 1998.

[16] A. J. Sarria, J. G. Lieber, S. K. Nordeen, and R. M. Evans, “The presence or absence of a vimentin-type intermediate filament network affects the shape of the nucleus in human SW-13 cells," Journal of Cell Science, vol. 107, no. part 6, pp. 1593-1607, 1994.

[17] S. D. Georgatos and G. Blobel, "Lamin B constitutes an intermediate filament attachment site at the nuclear envelope," Journal of Cell Biology, vol. 105, no. 1, pp. 117-125, 1987.

[18] R. Spurny, M. Gregor, M. J. Castañón, and G. Wiche, "Plectin deficiency affects precursor formation and dynamics of vimentin networks," Experimental Cell Research, vol. 314, no. 19, pp. 3570-3580, 2008.

[19] O. Esue, A. A. Carson, Y. Tseng, and D. Wirtz, "A direct interaction between actin and vimentin filaments mediated by the tail domain of vimentin," The Journal of Biological Chemistry, vol. 281, no. 41, pp. 30393-30399, 2006.

[20] S. Krüger, K. Sotlar, I. Kausch, and H.-P. Horny, "Expression of KIT (CD117) in renal cell carcinoma and renal oncocytoma," Oncology, vol. 68, no. 2-3, pp. 269-275, 2005.

[21] F. Lin, R. E. Brown, T. Shen, X. J. Yang, and C. Schuerch, "Immunohistochemical detection of P504S in primary and metastatic renal cell carcinomas," Applied Immunohistochemistry and Molecular Morphology, vol. 12, no. 2, pp. 153-159, 2004.

[22] V. Molinié, A. Balaton, S. Rotman et al., "Alpha-methyl CoA racemase expression in renal cell carcinomas," Human Pathology, vol. 37, no. 6, pp. 698-703, 2006. 


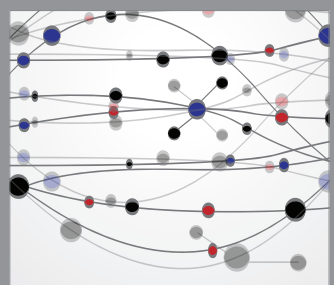

The Scientific World Journal
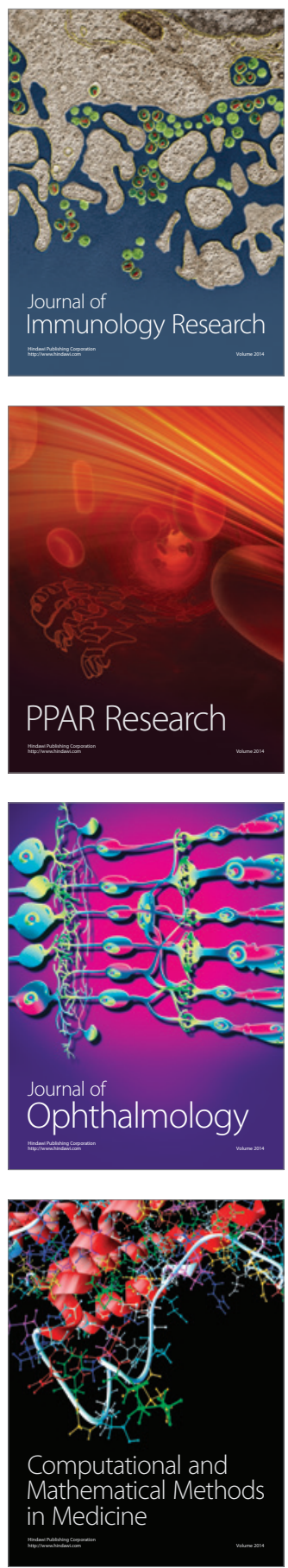

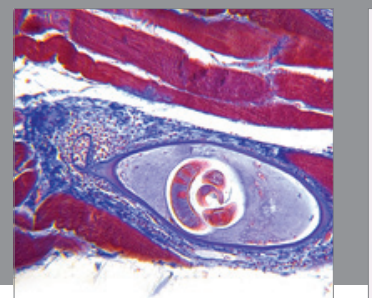

Gastroenterology

Research and Practice
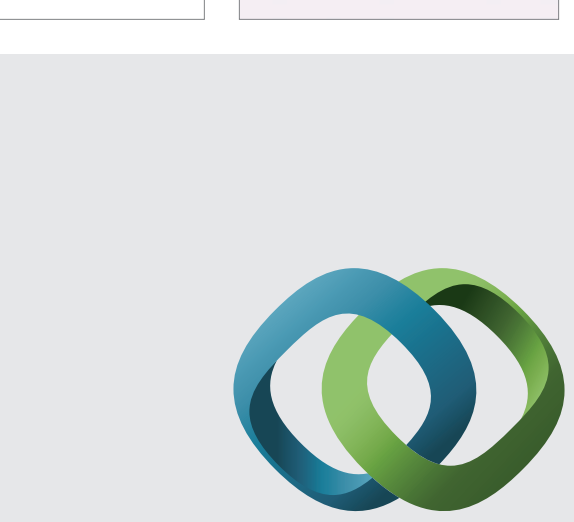

\section{Hindawi}

Submit your manuscripts at

http://www.hindawi.com
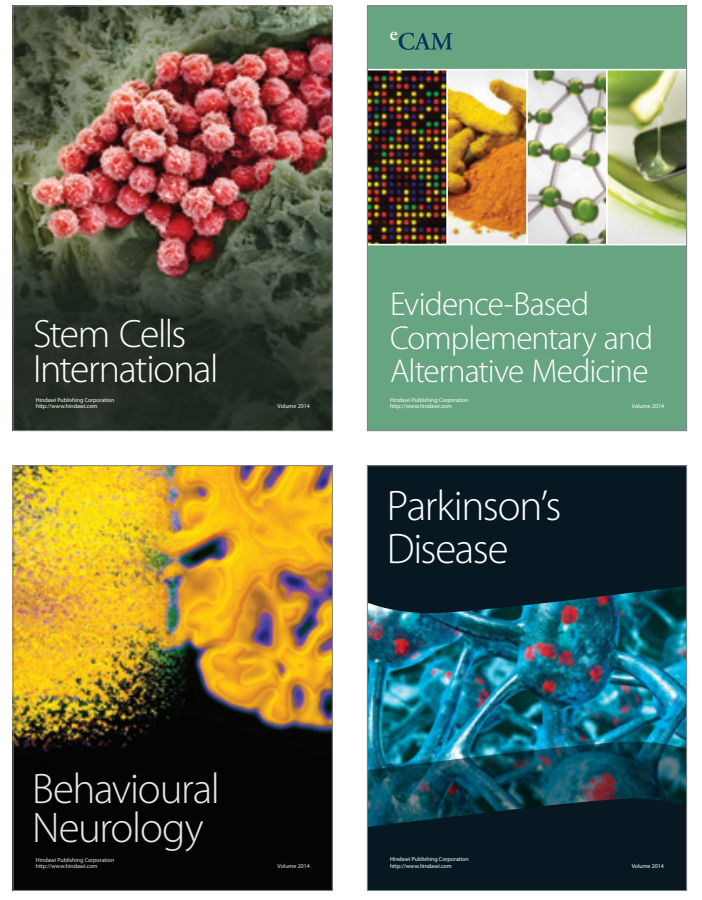
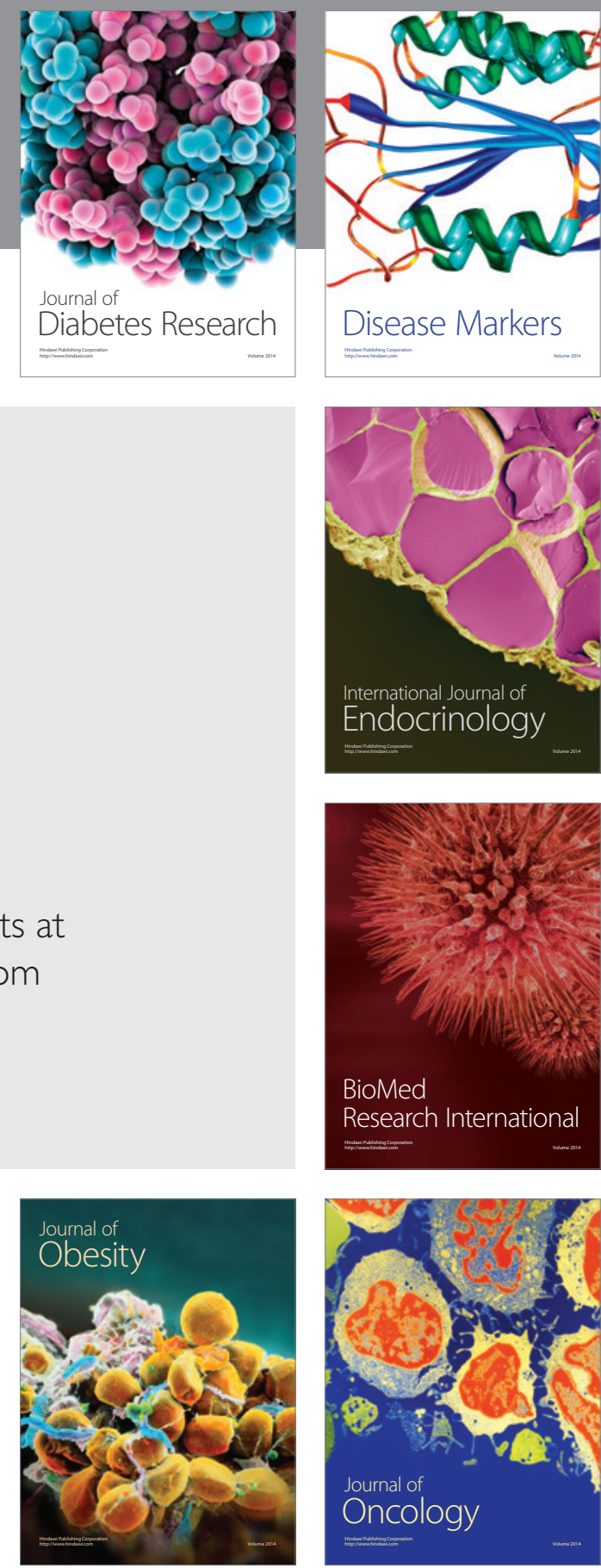

Disease Markers
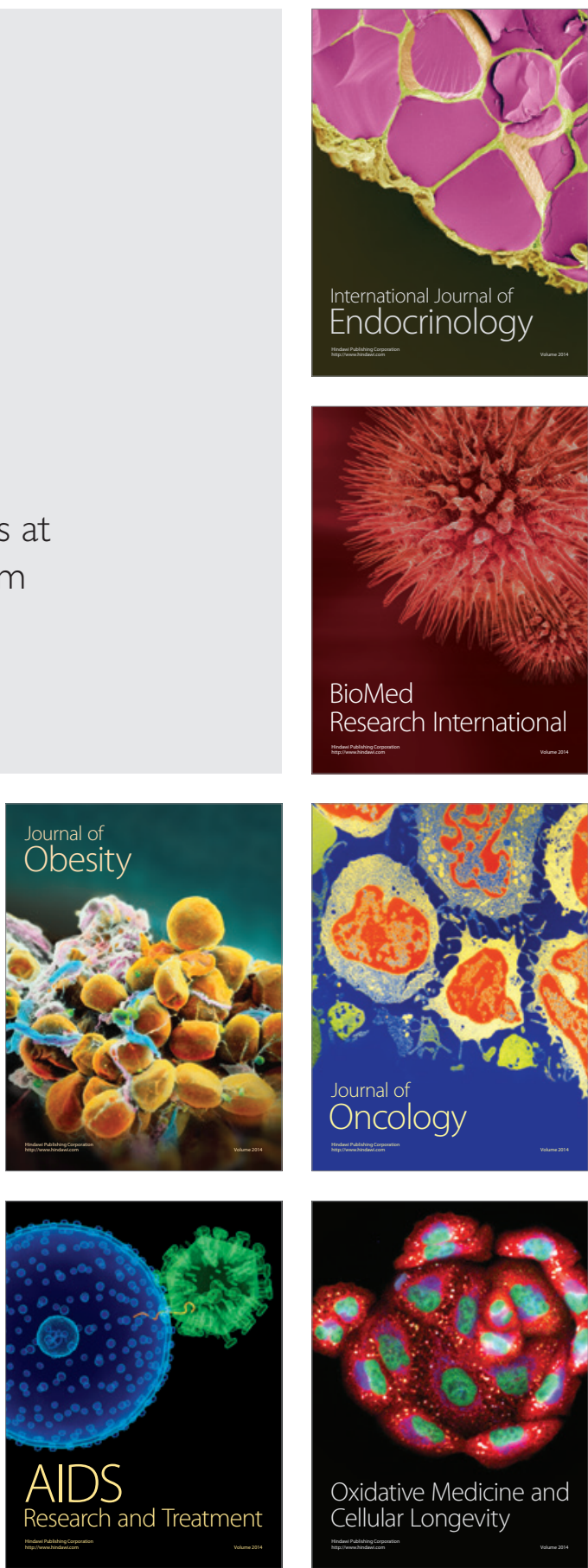